\title{
Postoperative Drainage with One Chest Tube Is Appropriate for Pulmonary Lobectomy: A Randomized Trial
}

\author{
Makoto Tanaka, ${ }^{1}$ Motoyasu Sagawa, ${ }^{1}$ Katsuo Usuda, ${ }^{1}$ Yuichiro Machida, ${ }^{1}$ \\ Masakatsu Ueno, ${ }^{1}$ Nozomu Motono ${ }^{1}$ and Tsutomu Sakuma ${ }^{1}$ \\ ${ }^{1}$ Department of Thoracic Surgery, Kanazawa Medical University, Uchinada, Kahoku, Ishikawa, Japan
}

\begin{abstract}
To expand postoperative residual lungs after pulmonary lobectomy, thoracic drainage with two chest tubes has been recommended. Several studies recently demonstrated that postoperative drainage with one chest tube (PD1) was as safe as that with two chest tubes (PD2). However, most of the patients in those studies underwent lobectomy by standard thoracotomy. Although the number of pulmonary lobectomies by video-assisted thoracic surgery (VATS) has been increasing in recent years, there have been no reports that compared PD1 with PD2 after pulmonary lobectomy, including that by VATS. To elucidate whether postoperative management with PD1 is as safe as that with PD2, we conducted a randomized controlled trial. Lung cancer patients who underwent lobectomies with mediastinal nodal dissection in our hospital were assigned to one of two groups: one chest tube placed in PD1 group and two chest tubes placed in PD2 group. A total of 108 patients were registered in the study. There were no significant differences in the age, gender, pathological stage or histological type between two groups. Since the residual lung expansion was good in both groups, there were no patients who needed thoracentesis. There were no significant differences in the number of cases with pleurodesis, the amount/duration of drainage or the pain of the patients between two groups. In conclusion, since PD1 has advantages in saving cost and time and in low risk of transcutaneous infection, PD1 is appropriate after pulmonary lobectomy by VATS and by open thoracotomy.
\end{abstract}

Keywords: chest tube drainage; lobectomy; operation; pain score; randomized trial Tohoku J. Exp. Med., 2014 January, 232 (1), 55-61. C 2014 Tohoku University Medical Press

\section{Introduction}

In order to expand postoperative residual lungs, textbooks recommend that two chest tubes should be placed after pulmonary lobectomy (Dexter and Kohman 1995; Martini and Ginsberg 2002; Shaw and LoCicero 2009). Usually, one chest tube is placed toward apex and another tube placed back to bottom. Air is drained from the apical drain, whereas blood and exudate are drained from the basal one. Theoretically, single tube chest drainage provides less pain and discomfort during drainage compared with double tube drainage, but single tube drainage has the possibility of inadequate chest drainage. Several randomized trials recently demonstrated that there was no significant difference in complications after lobectomy between patients with postoperative drainage using one chest tube (PD1) and patients with postoperative drainage using two chest tubes (PD2) (Gomez-Caro et al. 2006; Pawelczyk et al. 2007; Okur et al. 2009). However, most of the patients in these studies underwent lobectomy by standard thoracotomy. Recently, the number of pulmonary lobectomy by video- assisted thoracic surgery (VATS) has been increasing as less-invasive surgery especially in Japan (Ueda et al. 2008; Kuwano et al. 2012), and the skin incision used for the operation has been getting smaller (Sagawa et al. 2009). PD1 has been sometimes used after pulmonary lobectomy performed by VATS, but there have been no reports that have conclusively proven the appropriate number of chest tubes after VATS lobectomy.

Therefore, to determine whether PD1 is appropriate in patients after pulmonary lobectomy not only by open thoracotomy but also by VATS, we conducted a randomized controlled trial, and compared the necessity of thoracentesis, amount of drainage fluid, duration of drainage and pain between the patients with PD1 and PD2.

\section{Patients and Methods}

Study design

This study was a prospective randomized study to assess whether PD1 after pulmonary lobectomy would be inferior to PD2 or not (non-inferiority trial). This study was approved by the Institutional Review Board of Kanazawa Medical University in

Received July 26, 2013; revised and accepted January 9, 2014. Published online February 1, 2014; doi: 10.1620/tjem.232.55.

Correspondence: Makoto Tanaka, M.D., Department of Thoracic Surgery, Kanazawa Medical University, 1-1 Uchinada, Ishikawa 9200293, Japan.

e-mail: dr-ryo@kanazawa-med.ac.jp 
November 2008 and was registered in the University Hospital Medical Information Network Clinical Trial Registration (UMINCTR), Japan (registration number: UMIN000009661).

\section{Registration and eligibility criteria}

Pre-registration was performed before the patient's operation. The inclusion criteria for pre-registration were as follows: 1) patients who were scheduled to undergo a lobectomy or bilobectomy with mediastinal nodal dissection (dissection should be performed including at least $50 \%$ of the whole mediastinal area (The Japan Lung Cancer Society 2010)); 2) age from 20 to 85 years; and 3) written informed consent was provided.

The exclusion criteria were as follows: 1) patients who were scheduled to undergo a pneumonectomy and 2) perioperative massive air leakage or massive bleeding was anticipated.

Just before placing chest tube(s) for postoperative drainage, the final judgment for registration was performed. At this time, patients who did not actually undergo lobectomy or bilobectomy with mediastinal nodal dissection were excluded from this trial. In addition, patients in whom postoperative massive air leakage or massive bleeding was anticipated were also excluded. Finally, the remaining patients were fully registered.

\section{Random assignment and chest tube placing}

Randomization was carried out in the operating room just after full registration, using a block-randomization method with stratification of the affected side. The patients were assigned to one of two groups. In PD1 group, a single chest tube (straight 24Fr.; tough-sil thoracic drain, Sumitomo bakelite Co., Ltd., Tokyo, Japan) was placed basically from the back to the apex. For the patients in PD2 group, two chest tubes were placed basically from the back to bottom (curved 24Fr.; tough-sil thoracic drain, Sumitomo bakelite Co., Ltd., Tokyo, Japan) and from anterior to apex (straight 24Fr.; tough-sil thoracic drain, Sumitomo bakelite Co., Ltd., Tokyo, Japan).

\section{Postoperative management}

When there were no air leaks and the amount of drainage fluid was $200 \mathrm{ml} /$ day or less, the chest tube was removed. In PD2 group, one of the two tubes was sometimes removed several days before the removal of the other chest tube, and in that case, the physician in charge decided the day when the first chest tube was removed.

For analgesia, patient-control epidural anesthesia (PCEA) was used until removal of the chest tube(s). Additionally, a $25 \mathrm{mg}$ diclofenac sodium suppository (DS) and/or intramuscular injection of 15 $\mathrm{mg}$ of pentazocine plus $25 \mathrm{mg}$ of hydroxyzine $(\mathrm{PH})$ were administered when the patient needed them. Some oral analgesics were also administered based on the patient requirements.

Even after the removal of chest tube(s), chest roentgenograms of the patient were taken for a few weeks. When the expansion of the residual lung was determined to be insufficient at a conference of our department with blinded information regarding the group assignment, thoracentesis was performed.

\section{Endpoints}

The primary endpoint was the number of cases that required thoracentesis, which represented inadequate expansion of the residual lung. The secondary endpoints were the total amount of drainage fluid, duration of drainage, analgesia requirements and pain score evaluated by a visual analogue scale (VAS).
When a drug was infused into the thoracic cavity for pleurodesis, the volume of the drug was subtracted from the volume of drainage fluid. In order to eliminate the influence of prolonged drainage on air leakage with regard to the 'duration of drainage', we also evaluated the duration of drainage until the day when the amount of drainage fluid was less than $200 \mathrm{ml} /$ day.

The degree of pain experienced by each patient was evaluated by the maximum pain score from immediately after the operation until discharge, as well as the number of times analgesia was required (PCEA, DS and/or PH). In this analysis, some patients were excluded because their clinical records about pain score/analgesia were incomplete. Because both the amount of drainage fluid and pain of the patient might have been influenced by the length of the incision, these data were also analyzed with stratification by the length of incision $(\leq 8 \mathrm{~cm}$ and $>8 \mathrm{~cm})$.

\section{Sample size and statistical analysis}

PD1 is evidently more economical and time-saving than PD2. The postoperative pain might therefore be lower in patients with PD1 than in those with PD2. However, PD1 could lead to an increase in the inappropriate expansion of the residual lung and the number of cases requiring thoracentesis compared with PD2. We assumed that PD1 would be useful when fewer than $20 \%$ of patients with PD1 would require postoperative thoracentesis whereas $3 \%$ of patients with PD2 would require thoracentesis (non-inferiority trial). With an alpha error of $5 \%$ and a power of $80 \%$, the sample size was calculated to be 82 patients for both groups.

Categorical variables were compared using the chi square test, Fisher's exact probability test or the G test (William's correction). Average values were compared using Student's $t$-test. $P$ values $<0.05$ were regarded to be statistically significant. The calculations were performed using the StatMate software program (Version 3.18, ATMS, Tokyo).

\section{Results}

From January 2009 to March 2012, 136 patients were pre-registered with written informed consent. After the pulmonary resection in the operating room, we excluded the patients who underwent pneumonectomy, who underwent only wedge resection and who did not undergo mediastinal nodal dissection. Finally, 108 patients were fully registered and randomized in this study (Fig. 1).

The pathological stage was determined according to the TNM classification (ver. 7). There was no statistically significant difference in the age, gender, pathological stage or histological type between the two groups (Table 1).

Since the residual lung expansion was appropriate in both groups, there were no patients who needed thoracentesis. There were no statistically significant differences in the number of cases requiring pleurodesis, the amount/duration of drainage and the pain of the patients between the two groups (Table 2).

Because the length of the incision might influence the results, a sub-analysis was performed after stratifying the patients by the length of incision $(\leq 8 \mathrm{~cm}$ and $>8 \mathrm{~cm}$ ) (Table 3). Even with stratification by length of incision, there were no statistically significant differences between 


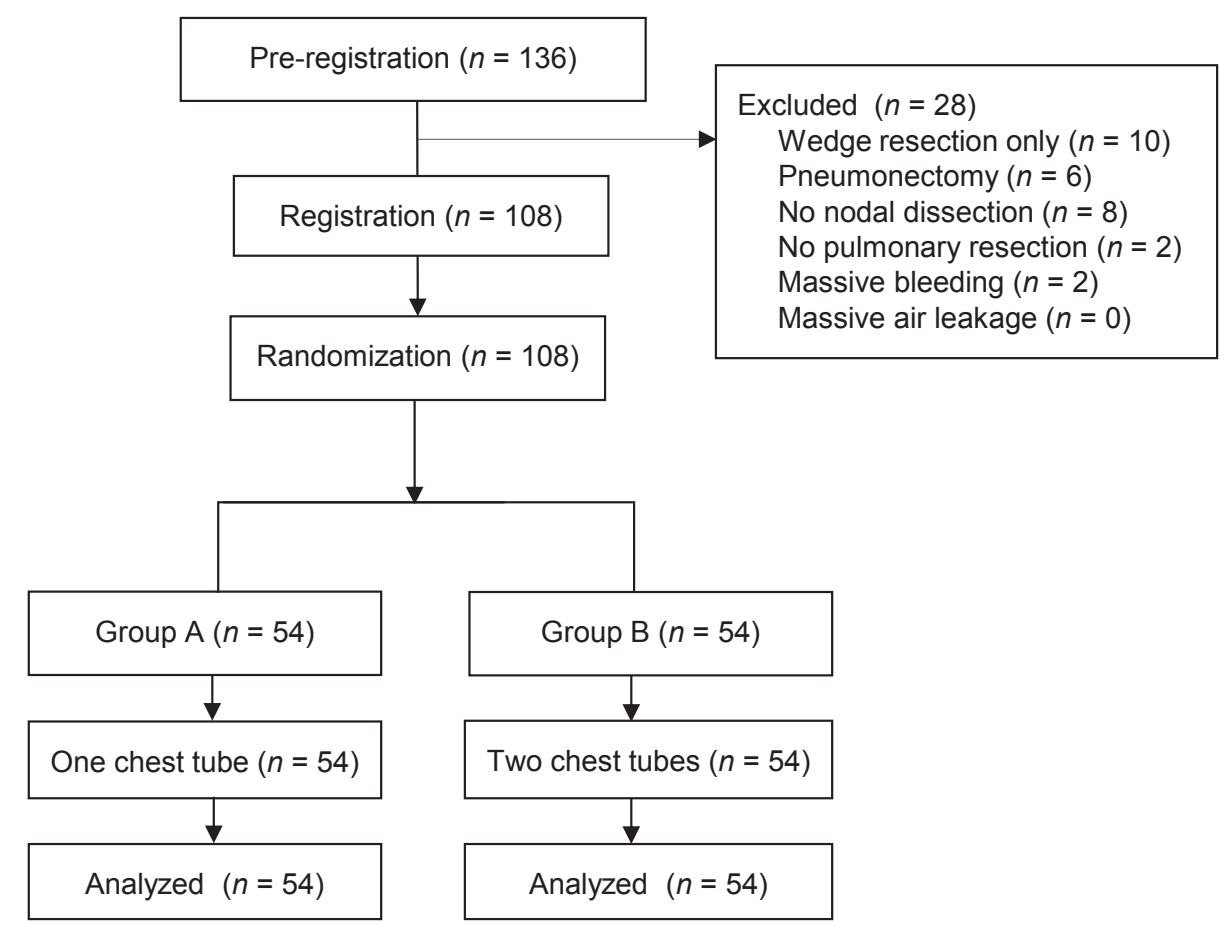

Fig. 1. The CONSORT (Consolidated Standards of Reporting Trials) diagram. The disposition of all participants is shown.

the two groups.

Another analysis was conducted to evaluate the relationship between the length of incision and drainage/pain, combining the two groups (Table 4). There were no significant differences in the amount or duration of drainage based on the length of the incision. Although the maximum pain scores of the patients with smaller incisions were lower than those of patients with larger incisions, the various analgesia requirements were not significantly different based on the length of the incision.

\section{Discussion}

The inappropriate extension of the residual lungs after the lobectomy is one of the most important causes of complications after surgery. Adequate re-expansion is an important factor that helps to avoid hypoxemia, atelectasis and acute respiratory insufficiency. Therefore, the conventional method of thoracic drainage after pulmonary lobectomy was to place two chest tubes; one drain in the apical position for the drainage of air and the other in the basal position for the drainage of blood and exudate (Dexter and Kohman 1995; Martini and Ginsberg 2002; Shaw and LoCicero 2009).

In recent years, many thoracic surgeons have adopted thoracic drainage after pulmonary lobectomy using only one chest tube. Several reports have been published comparing the use of one chest tube with the use of two chest tubes for thoracic drainage after pulmonary lobectomy, but most of these were observational studies (Alex et al. 2003; Kejriwal and Newman 2005; Icard et al. 2006), and there have so far only been three reports of randomized controlled trials (Gomez-Caro et al. 2006; Pawelczyk et al. 2007; Okur et al. 2009). The results of the randomized trials revealed that proper extension of the residual lung could be achieved even with one chest tube. These reports also revealed that there were no significant differences in the postoperative complications, whereas the overall costs were clearly reduced (Gomez-Caro et al. 2006; Pawelczyk et al. 2007; Okur et al. 2009). However, most of the patients in these trials underwent pulmonary lobectomy using standard thoracotomy, therefore, these results cannot be fully applied to the patients treated by VATS, which is becoming mainstream for pulmonary surgery, especially in Japan (Ueda et al. 2008; Kuwano et al. 2012). In this study, approximate the half of the patients had skin incision of $8 \mathrm{~cm}$ or less, and they were regarded as treated by VATS. The present report is the first report of a randomized trial to assess the appropriate number of postoperative chest tubes where the patients in the trial consisted of those who underwent pulmonary lobectomy by VATS as well as by open thoracotomy.

We randomly assigned 108 participants to either PD1 or PD2. There were no significant differences in the number of cases requiring thoracentesis ( 0 in both groups), the amount/duration of drainage or the maximum pain score between the two groups, which meant that PD1 was not inferior to PD2. Therefore, due to the lack of inferiority, and the advantages in saving cost and time to place and remove the second chest tube as well as in decreasing the risk of transcutaneous infection, PD1 should therefore be 
Table 1. The characteristics of the patients.

\begin{tabular}{|c|c|c|c|c|}
\hline & $\begin{array}{l}\text { PD1 group } \\
(n=54)\end{array}$ & $\begin{array}{l}\text { PD2 group } \\
(n=54)\end{array}$ & $\begin{array}{c}\text { Total } \\
(n=108)\end{array}$ & $P$ value \\
\hline Age (mean \pm s.D.) & $66.8 \pm 7.5$ & $67.7 \pm 8.0$ & $67.3 \pm 7.7$ & 0.527 \\
\hline Sex (Male / Female) & $38 / 16$ & $32 / 22$ & $70 / 38$ & 0.227 \\
\hline Operative side (Right / Left) & $30 / 24$ & $30 / 24$ & $60 / 48$ & 1.000 \\
\hline Incision length & & & & 0.848 \\
\hline$\leq 8 \mathrm{~cm}$ & 27 & 28 & 55 & \\
\hline$>8 \mathrm{~cm}$ & 27 & 26 & 53 & \\
\hline Resected lobe & & & & 0.892 \\
\hline Upper & 23 & 20 & 43 & \\
\hline Lower & 23 & 27 & 50 & \\
\hline Middle & 2 & 2 & 4 & \\
\hline Bilobectomy (or lobectomy + partial resection) & 6 & 5 & 11 & \\
\hline Histology & & & & 0.322 \\
\hline Adenocarcinoma & 35 & 39 & 74 & \\
\hline Squamous cell carcinoma & 9 & 12 & 21 & \\
\hline Small cell carcinoma & 2 & 1 & 3 & \\
\hline Combined/double lung cancer & 2 & 2 & 4 & \\
\hline Large cell carcinoma & 3 & 0 & 3 & \\
\hline Metastatic lung cancer & 2 & 0 & 2 & \\
\hline Sarcoma & 1 & 0 & 1 & \\
\hline Pathological stage (except metastatic lung cancer) & & & & 0.830 \\
\hline Stage IA & 19 & 28 & 47 & \\
\hline Stage IB & 15 & 10 & 25 & \\
\hline Stage IIA & 7 & 6 & 13 & \\
\hline Stage IIB & 3 & 2 & 5 & \\
\hline Stage IIIA & 6 & 6 & 12 & \\
\hline Stage IIIB & 0 & 0 & 0 & \\
\hline Stage IV & 2 & 2 & 4 & \\
\hline $\mathrm{T}$ factor (except metastatic lung cancer) & & & & 0.370 \\
\hline $\mathrm{T} 1 \mathrm{a} / \mathrm{T} 1 \mathrm{~b}$ & 19 & 26 & 45 & \\
\hline $\mathrm{T} 2 \mathrm{a} / \mathrm{T} 2 \mathrm{~b}$ & 28 & 21 & 49 & \\
\hline $\mathrm{T} 3$ & 5 & 6 & 11 & \\
\hline $\mathrm{T} 4$ & 0 & 1 & 1 & \\
\hline $\mathrm{N}$ factor (except metastatic lung cancer) & & & & 0.121 \\
\hline N0 & 39 & 46 & 85 & \\
\hline N1 & 8 & 2 & 10 & \\
\hline $\mathrm{N} 2$ & 5 & 6 & 11 & \\
\hline M factor (except metastatic lung cancer) & & & & 0.523 \\
\hline M0 & 50 & 52 & 102 & \\
\hline M1a & 2 & 1 & 3 & \\
\hline M1b & 0 & 1 & 1 & \\
\hline
\end{tabular}

adopted instead of PD2. Of note, these results were not influenced by the length of the incision.

There have been three randomized trials concerning the differences in the outcome based on the number of chest tubes placed after pulmonary lobectomy (Gomez-Caro et al. 2006; Pawelczyk et al. 2007; Okur et al. 2009). The amount of drainage were reported to decrease in the patients treated with PD1 in one study (Okur et al. 2009), but there were no statistically significant differences between PD1 and PD2 in two other reports (Gomez-Caro et al. 2006; Pawelczyk et al. 2007). The duration of drainage was shorter with PD1 in one report (different from the above 
Table 2. Thoracentesis, drainage fluid, analgesia requirements and pain score.

\begin{tabular}{|c|c|c|c|c|}
\hline & & PD1 group & PD2 group & $P$ value \\
\hline \multirow[t]{2}{*}{ Thoracentesis } & $(+)$ & 0 & 0 & 1.000 \\
\hline & $(-)$ & 54 & 54 & \\
\hline \multirow[t]{2}{*}{ Pleurodesis } & $(+)$ & 1 & 4 & 0.117 \\
\hline & $(-)$ & 53 & 50 & \\
\hline \multicolumn{2}{|c|}{ Amount of drainage (ml) } & $789.2 \pm 607.6$ & $913.2 \pm 960.9$ & 0.424 \\
\hline \multicolumn{2}{|c|}{ Duration of drainage (days) } & $3.78 \pm 2.16$ & $4.61 \pm 5.74$ & 0.321 \\
\hline \multicolumn{2}{|c|}{ Duration until 200* (days) } & $2.39 \pm 1.85$ & $1.98 \pm 1.16$ & 0.173 \\
\hline \multicolumn{2}{|c|}{ PCEA** (times) } & $6.78 \pm 10.31(n=50)$ & $7.18 \pm 11.87(n=50)$ & 0.875 \\
\hline \multicolumn{2}{|c|}{ DS*** (times) } & $0.56 \pm 0.86$ & $1.13 \pm 3.13$ & 0.196 \\
\hline \multicolumn{2}{|c|}{$\mathrm{PH}^{* * * *}$ (times) } & $1.46 \pm 2.72$ & $2.17 \pm 3.45$ & 0.241 \\
\hline \multicolumn{2}{|c|}{ Maximum pain score } & $4.57 \pm 3.23(n=44)$ & $3.92 \pm 3.08(n=36)$ & 0.362 \\
\hline
\end{tabular}

Duration until 200*: duration of drainage until the amount of drainage fluid was less than $200 \mathrm{ml} /$ day.

PCEA**: patient-control epidural anesthesia.

DS***: $25 \mathrm{mg}$ of diclofenac sodium suppository.

$\mathrm{PH}^{* * * *}$ : intramuscular injection of $15 \mathrm{mg}$ of pentazocine plus $25 \mathrm{mg}$ of hydroxyzine.

Table 3. Drainage fluid, analgesia requirements and pain score stratified by the length of incision and group.

\begin{tabular}{lcccc}
\hline & Length of incision & PD1 group & PD2 group & $P$ value \\
\hline Amount of drainage (ml) & $\leq 8 \mathrm{~cm}$ & $622.6 \pm 465.6(n=27)$ & $944.4 \pm 1,266.9(n=28)$ & 0.220 \\
& $>8 \mathrm{~cm}$ & $955.9 \pm 691.4(n=27)$ & $879.7 \pm 470.7(n=26)$ & 0.642 \\
Duration of drainage (days) & $\leq 8 \mathrm{~cm}$ & $3.41 \pm 2.04(n=27)$ & $4.21 \pm 2.30(n=28)$ & 0.194 \\
& $>8 \mathrm{~cm}$ & $4.15 \pm 2.25(n=27)$ & $4.58 \pm 4.06(n=26)$ & 0.635 \\
Duration until 200* (days) & $\leq 8 \mathrm{~cm}$ & $2.04 \pm 1.40(n=27)$ & $2.00 \pm 1.08(n=28)$ & 0.913 \\
& $>8 \mathrm{~cm}$ & $2.74 \pm 2.18(n=27)$ & $1.96 \pm 1.25(n=26)$ & 0.118 \\
\hline PCEA** (times) & $\leq 8 \mathrm{~cm}$ & $6.80 \pm 11.50(n=25)$ & $7.80 \pm 14.25(n=25)$ & 0.786 \\
& $>8 \mathrm{~cm}$ & $6.76 \pm 9.21(n=25)$ & $6.56 \pm 9.17(n=25)$ & 0.939 \\
DS*** (times) & $\leq 8 \mathrm{~cm}$ & $0.67 \pm 0.92(n=27)$ & $0.61 \pm 1.42(n=28)$ & 0.855 \\
PH**** (times) & $>8 \mathrm{~cm}$ & $0.44 \pm 0.80(n=27)$ & $1.69 \pm 4.23(n=26)$ & 0.138 \\
& $\leq 8 \mathrm{~cm}$ & $1.89 \pm 3.59(n=27)$ & $2.00 \pm 3.23(n=28)$ & 0.904 \\
Maximum pain score & $>8 \mathrm{~cm}$ & $1.04 \pm 1.81(n=27)$ & $2.35 \pm 3.73(n=26)$ & 0.092 \\
& $\leq 8 \mathrm{~cm}$ & $4.35 \pm 3.47(n=20)$ & $2.74 \pm 2.51(n=19)$ \\
\hline
\end{tabular}

Duration until 200*: duration of drainage until the amount of drainage fluid was less than $200 \mathrm{ml} /$ day.

PCEA**: patient-control epidural anesthesia.

DS***: $25 \mathrm{mg}$ of diclofenac sodium suppository.

$\mathrm{PH}^{* * * *}$ : intramuscular injection of $15 \mathrm{mg}$ of pentazocine plus $25 \mathrm{mg}$ of hydroxyzine.

report with a smaller amount of drainage with PD1) (Pawelczyk et al. 2007), but the remaining reports revealed no significant differences between the groups (Gomez-Caro et al. 2006; Okur et al. 2009). All three reports showed that no significant differences existed concerning the requirements for additional chest tube drainage (Gomez-Caro et al. 2006; Pawelczyk et al. 2007; Okur et al. 2009). In the present study, there were no significant differences in the amount/duration of drainage or need for additional thoracentesis between PD1 and PD2, which were in agreement with the results of the majority of these three randomized trials.

In order to assess the patients' pain, two randomized trials reported the pain scores of the patients (Pawelczyk et al. 2007; Okur et al. 2009). One of them stated that, although the pain scores of the patients on the fourth postoperative day were lower with PD1 than PD2, the pain scores were not significantly different on the first, second or third postoperative day (Pawelczyk et al. 2007). The other report showed that the pain scores of the patients on the second and fourteenth postoperative days were lower with PD1 than PD2 (Okur et al. 2009). One of the reasons of the 
Table 4. Drainage fluid, analgesia requirements and pain score in the overall population according to the length of incision.

\begin{tabular}{|c|c|c|c|}
\hline & $\begin{array}{l}\text { Incision length } \leq 8 \mathrm{~cm} \\
(n=55)\end{array}$ & $\begin{array}{l}\text { Incision length }>8 \mathrm{~cm} \\
\quad(n=53)\end{array}$ & $P$ value \\
\hline Amount of drainage (ml) & $786.4 \pm 966.1$ & $918.5 \pm 589.1$ & 0.395 \\
\hline Duration of drainage (days) & $4.04 \pm 5.21$ & $4.36 \pm 3.24$ & 0.701 \\
\hline Duration until 200* (days) & $2.02 \pm 1.24$ & $2.36 \pm 1.81$ & 0.255 \\
\hline PCEA** (times) & $7.30 \pm 12.83(n=50)$ & $6.66 \pm 9.09(n=50)$ & 0.774 \\
\hline DS*** (times) & $0.64 \pm 1.19$ & $1.06 \pm 3.05$ & 0.345 \\
\hline $\mathrm{PH}^{* * * *}$ (times) & $1.95 \pm 3.38$ & $1.68 \pm 2.83$ & 0.659 \\
\hline Maximum pain score & $3.56 \pm 3.11(n=39)$ & $4.95 \pm 3.10(n=41)$ & 0.049 \\
\hline
\end{tabular}

Duration until 200*: duration of drainage until the amount of drainage fluid was less than $200 \mathrm{ml} /$ day.

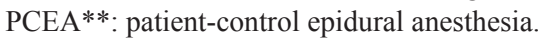

DS***: $25 \mathrm{mg}$ of diclofenac sodium suppository.

$\mathrm{PH}^{* * * *}$ : intramuscular injection of $15 \mathrm{mg}$ of pentazocine plus $25 \mathrm{mg}$ of hydroxyzine.

difference between the results of these two reports might be the difference in types of analgesia adopted in these studies. The analgesia requirements were also evaluated in two randomized trials, where there was some tendency for less analgesia to be required with PD1 than with PD2 (GomezCaro et al. 2006; Pawelczyk et al. 2007). In the present study, both the pain scores and analgesia requirements were not significantly different between the patients treated with PD1 and PD2. In our study, epidural analgesia was administered until the removal of chest tubes. Oral analgesics were not restricted and were sufficiently added when the patients desired them, because we believe that the appropriate relief of postoperative pain is an important right of the patients. Furthermore, the length of the skin incision in the patients included in this trial may have been smaller than that of other studies. All of these factors might have minimized the difference between PD1 and PD2.

We additionally evaluated the amount/duration of drainage fluid, analgesia requirements and pain score according to the length of incision, combining the two groups. Although the maximum pain score was lower in patients with a smaller skin incision, the amount/duration of drainage fluid and analgesia requirements were not significantly different based on the length of the skin incision, which meant that the advantage of a smaller skin incision was limited.

There are some issues to be discussed regarding this trial. First, in the PD2 group, one of the two chest tubes was sometimes removed early for various reasons. However, the removal of one of the two tubes did not seem to cause any disadvantages for the patients in the PD2 group. Second, in our study, several different oral analgesics were used, which made the assessment of patients' pain more difficult. However, as described above, the relief of the pain was a very important issue for the patients, and the patient response to analgesia differed, so we did not know whichever agent was the most suitable for the particular patient. Therefore, we permitted the flexible use of analgesia, but this point was one of the limitations of this study.
Third, although the pain scores of patients on certain postoperative days were adopted in other papers, we used the 'maximum pain scores' in our study. According to our experience, the time when a patient experiences the maximum degree of pain varies. Therefore, we assumed that the maximum pain scores, regardless of the postoperative day, were the most suitable information to best assess the patients' pain.

In conclusion, the difference in the number of chest tubes was not associated with any significant difference in the expansion of the residual lungs, the amount/duration of drainage fluid or the patients' pain in the patients after pulmonary lobectomy by VATS or open thoracotomy without massive bleeding/air-leak. Therefore, PD1 is considered to be appropriate for the post-lobectomy chest drainage of such patients.

\section{Acknowledgements}

We are grateful to Dr. Koshi Nakamura (Department of Epidemiology and Public Health, Kanazawa Medical University) for the statistical analysis of this trial.

This study was supported in part by Grants-in-Aid for Scientific Research from the Ministry of Education, Culture, Sports, Science and Technology, Japan (No. 25670610).

\section{Conflict of Interest}

The authors declare no conflict of interest.

\section{References}

Alex, J., Ansari, J., Bahalkar, P., Agarwala, S., Rehman, M.U., Saleh, A. \& Cowen, M.E. (2003) Comparison of the immediate postoperative outcome of using the conventional two drains versus a single drain after lobectomy. Ann. Thorac. Surg., 76, 1046-1049.

Dexter, E.U. \& Kohman, L. (1995) Perioperative care of patients undergoing thoracic surgery. In Surgery of the chest. Volume 1, 7th ed., edited by Sabiston, D.C. \& Spencer, F. W.B. Saunders, Philadelphia, PA, pp. 43-57.

Gomez-Caro, A., Roca, M.J., Torres, J., Cascales, P., Terol, E., Castaner, J., Pinero, A. \& Parrilla, P. (2006) Successful use of a single chest drain postlobectomy instead of two classical drains: a randomized study. Eur. J. Cardiothorac. Surg., 29, 
562-566.

Icard, P., Chautard, J., Zhang, X.D., Juanico, M., Bichi, S., Lerochais, J.P. \& Flais, F. (2006) A single 24F Blake drain after wedge resection or lobectomy: a study on 100 consecutive cases. Eur. J. Cardiothorac. Surg., 30, 649-651.

Kejriwal, N.K. \& Newman, M.A.J. (2005) Use of a single silastic chest drain following thoracotomy: initial evaluation. ANZ J. Surg., 75, 710-712.

Kuwano, H., Amano, J. \& Yokomise, H. (2012) Thoracic and cardiovascular surgery in Japan during 2010: annual report by The Japanese Association for Thoracic Surgery. Gen. Thorac. Cardiovasc. Surg., 60, 680-708.

Martini, N. \& Ginsberg, R.J. (2002) Lobectomy. In Thoracic Surgery, edited by Pearson, F.G., Deslauriers, J., Ginsberg, R.J., Hiebert, C.A., McKneally, M.F. \& Urschel, H.C. Churchill Livingstone, New York, NY, pp. 981-990.

Okur, E., Baysungur, V., Tezel, C., Sevilgen, G., Ergene, G., Gokce, M. \& Halezeroglu, S. (2009) Comparison of the single or double chest tube applications after pulmonary lobectomies. Eur. J. Cardiothorac. Surg., 35, 32-36.

Pawelczyk, K., Marciniak, M., Kacprzak, G. \& Kolodziej, J.
(2007) One or two drains after lobectomy? A comparison of both methods in the immediate postoperative period. Thorac. Cardiovasc. Surg., 55, 313-316.

Sagawa, M., Higashi, K., Usuda, K., Aikawa, H., Machida, Y., Tanaka, M., Ueno, M. \& Sakuma, T. (2009) Curative wedge resection for non-invasive bronchioloalveolar carcinoma. Tohoku J. Exp. Med., 217, 133-137.

Shaw, J.P. \& LoCicero, III. J. (2009) Postoperative management of the general thoracic surgical patient. In General thoracic surgery, 7th ed., edited by Shields, T.W., LoCicero, III. J., Reed, C.E. \& Feins, R.H. Lippincott Williams \& Wilkins, Philadelphia, PA, pp. 571-579.

The Japan Lung Cancer Society (2010) The Manual of Documenting the Lung Cancer Surgery. In General rule for clinical and pathological record of lung cancer, 7th ed., Kanehara Shuppan, Tokyo, Japan, pp. 47-61.

Ueda, Y., Fujii, Y. \& Udagawa, H. (2008) Thoracic and cardiovascular surgery in Japan during 2006: annual report by the Japanese Association for Thoracic Surgery. Gen. Thorac. Cardiovasc. Surg., 56, 365-388. 